\title{
Reconfiguring 10-colourings of planar graphs
}

\author{
Carl Feghali \\ Department of Informatics, \\ University of Bergen, \\ Bergen, Norway \\ carl.feghali@uib.no
}

\begin{abstract}
Let $k \geq 1$ be an integer. The reconfiguration graph $R_{k}(G)$ of the $k$-colourings of a graph $G$ has as vertex set the set of all possible $k$-colourings of $G$ and two colourings are adjacent if they differ on exactly one vertex.

A conjecture of Cereceda from 2007 asserts that for every integer $\ell \geq k+2$ and $k$-degenerate graph $G$ on $n$ vertices, $R_{\ell}(G)$ has diameter $O\left(n^{2}\right)$. The conjecture has been verified only when $\ell \geq 2 k+1$. We give a simple proof that if $G$ is a planar graph on $n$ vertices, then $R_{10}(G)$ has diameter at most $n^{2}$. Since planar graphs are 5-degenerate, this affirms Cereceda's conjecture for planar graphs in the case $\ell=2 k$.
\end{abstract}

Let $k \geq 1$ be an integer. The reconfiguration graph $R_{k}(G)$ of the $k$ colourings of a graph $G$ has as vertex set the set of all possible $k$-colourings of $G$ and two colourings are adjacent if they differ on the colour of exactly one vertex of $G$. A list assignment of a graph is a function $L$ that assigns to each vertex $v$ a list $L(v)$ of colours. The graph $G$ is $L$-colourable if it has a proper colouring $f$ such that $f(v) \in L(v)$ for each vertex $v$ of $G$.

For a positive integer $d$, a graph $G$ is $d$-degenerate if every subgraph of $G$ contains a vertex of degree at most $d$. Expressed in another way, $G$ is $d$-degenerate if there there exists an ordering $v_{1}, \ldots, v_{n}$ of the vertices in $G$ such that each $v_{i}$ has at most $d$ neighbours $v_{j}$ with $j<i$.

Reconfiguration problems have received much attention in the past decade; we refer the reader to the surveys by van den Heuvel [12] and Nishimura [9]. 
In this note, we are concerned with a conjecture of Cereceda [3] from 2007 which asserts that for every integer $\ell \geq k+2$ and $k$-degenerate graph $G$ on $n$ vertices, $R_{\ell}(G)$ has diameter $O\left(n^{2}\right)$. Cereceda [3] verified the conjecture whenever $\ell \geq 2 k+1$ but the conjecture remains open for every other value $2 k \geq \ell \geq k+2$. It is also known to hold for graphs of bounded tree-width [1] (the claimed shorter proof in [6] yields instead a bound of $O\left(t n^{2}\right)$ on the diameter, where $t$ is the tree-width of the graph under consideration) as well as $(\Delta-1)$-degenerate graphs [8], where $\Delta$ is the maximum degree of the graph under consideration. Our aim in this note is to address the conjecture for planar graphs in the following theorem.

Theorem 1. For every planar graph $G$ on $n$ vertices, $R_{10}(G)$ has diameter at most $n^{2}$.

Since planar graphs are 5-degenerate, Theorem 1 affirms Cereceda's conjecture for planar graphs in the case $\ell=2 k$. In all other cases, some partial results are known. Given a planar graph $G$ on $n$ vertices, it is shown in [2] that $R_{\ell}(G)$ has diameter $O\left(n^{c}\right)$ for each $\ell \geq 8$ and some (large) positive constant $c$ (see [7] for a short proof of this result with a weaker bound on $c$ ) while in [5] it is shown that $R_{7}(G)$ has diameter $2^{O(\sqrt{n})}$.

Let us note that the novelty of our approach lies, in some sense, on a new trick that essentially reformulates the reconfiguration problem as a list colouring problem. In particular, Theorem 1 will follow as a corollary from the following special case of a famous theorem due to Thomassen [10].

Theorem 2. Let $G$ be a planar graph, and let $v$ be a vertex of $G$. Suppose that $L(u)$ is a list of one colour if $u=v$ and a list of at least five colours if $u \in V(G)-\{v\}$. Then $G$ is L-colourable.

Proof of Theorem 11. Since $G$ is 5-degenerate, we can order the vertices of $G$ as $v_{1}, \ldots, v_{n}$ such that each $v_{i}$ has at most five neighbours $v_{j}$ with $j<i$.

Let $\alpha$ and $\beta$ be 10-colourings of $G$, and let $h$ be the lowest index such that $\alpha\left(v_{h}\right) \neq \beta\left(v_{h}\right)$. Starting from $\alpha$, we shall describe a sequence of recolourings such that

- for $i<h, v_{i}$ is not recoloured,

- for $i>h, v_{i}$ is recoloured at most once, and

- $v_{h}$ is recoloured with colour $\beta\left(v_{h}\right)$. 
By repeatedly using such sequences, we can recolour $\alpha$ to $\beta$ by at most $n$ recolourings per vertex and the theorem follows.

To describe the sequence, let $H$ be the graph induced by $S=\left\{v_{h}, \ldots, v_{n}\right\}$. We start by finding a list assignment $L$ of $H$ as follows:

- $L\left(v_{h}\right)=\left\{\beta\left(v_{h}\right)\right\}$, and

- for $i>h, L\left(v_{i}\right)=\{1, \ldots, 10\} \backslash\left\{\alpha\left(v_{j}\right):\left(v_{i}, v_{j}\right) \in E(G), j<i\right\}$.

Applying Theorem 2, we obtain an $L$-colouring $f$ of $H$. We then simply recolour $v_{k}$ with $f\left(v_{k}\right)$ starting with $v_{n}$ and working backwards through $S$. (It is possible that $f\left(v_{k}\right)=\alpha\left(v_{k}\right)$ in which case the colour of $v_{k}$ is unchanged.) Each colouring obtained is proper since $v_{n}$ has no neighbours coloured $f\left(v_{n}\right)$ and when a vertex $v_{k}, k<n$, is recoloured, its neighbours $v_{j}$ with $j<k$ do not have colour $f\left(v_{k}\right)$ by definition of $L\left(v_{k}\right)$ nor do its other neighbours $v_{j}$ with $j>k$ since $f$ is a proper colouring. Given that, at the end of the sequence, $v_{h}$ is recoloured to colour $\beta\left(v_{h}\right)$, this completes the proof.

It is not difficult to prove the following theorem using the same approach as in the proof of Theorem 1 .

Theorem 3. Let $k$ and $\ell$ be positive integers, let $G$ be a $k$-degenerate graph on $n$ vertices, and let $v$ be a vertex of $G$. Suppose that $L(u)$ is a list of one colour if $u=v$ and a list of at least $\ell$ colours if $u \in V(G)-\{v\}$. If $G$ is $L$-colourable, then $R_{k+\ell}(G)$ has diameter at most $n^{2}$.

We state two of possibly other consequences of Theorem 3 .

Corollary 1. Let $G$ be a planar graph on $n$ vertices and of girth 5 . Then $R_{6}(G)$ has diameter at most $n^{2}$.

Proof. Since planar graphs of girth 5 are 3 -degenerate, the result is immediate from Theorem 3 combined with Theorem 2.1 in [11].

Corollary 2. Let $k$ be a positive integer, and let $G$ be a $k$-degenerate graph on $n$ vertices. If $k+1$ is prime, then $R_{2 k+1}(G)$ has diameter at most $n^{2}$.

Proof. Combine Theorem 3 with Theorem 6 in 4 . 


\section{Acknowledgements}

The author is grateful to Louis Esperet for pointing out Corollary 2. This work is supported by the Research Council of Norway via the project CLASSIS. It was conducted while the author was visiting the Department of Applied Mathematics of the Faculty of Mathematics and Physics at Charles University.

\section{References}

[1] M. Bonamy and N. Bousquet. Recoloring graphs via tree decompositions. European Journal of Combinatorics, 69:200-213, 2018.

[2] N. Bousquet and G. Perarnau. Fast recoloring of sparse graphs. European Journal of Combinatorics, 52:1-11, 2016.

[3] L. Cereceda. Mixing graph colourings. PhD thesis, London School of Economics, 2007.

[4] Z. Dvořák, S. Norin, and L. Postle. List coloring with requests. Journal of Graph Theory, 2016.

[5] E. Eiben and C. Feghali. Towards Cereceda's conjecture for planar graphs. arXiv, 1810.00731, 2018.

[6] C. Feghali. Paths between colourings of graphs with bounded tree-width. Information Processing Letters, 2018.

[7] C. Feghali. Paths between colourings of sparse graphs. European Journal of Combinatorics, 75:169-171, 2019.

[8] C. Feghali, M. Johnson, and D. Paulusma. A reconfigurations analogue of Brooks' theorem and its consequences. Journal of Graph Theory, 83(4):340-358, 2016.

[9] N. Nishimura. Introduction to reconfiguration. Algorithms, 11(4):52, 2018.

[10] C. Thomassen. Every planar graph is 5-choosable. Journal of Combinatorial Theory Series B, 62(1):180-181, 1994. 
[11] C. Thomassen. 3-list-coloring planar graphs of girth 5. Journal of Combinatorial Theory Series B, 64(1):101-107, 1995.

[12] J. van den Heuvel. The complexity of change. Surveys in Combinatorics 2013, edited by S. R. Blackburn, S. Gerke, and M. Wildon, London Mathematical Society Lecture Notes Series, 409, 2013. 